\title{
Effect of Interband Transitions on $c$-axis Penetration Depth of Layered Superconductors
}

\author{
W. A. Atkinsonti and J. P. Carbotte \\ Department of Physics and Astronomy, McMaster University, Hamilton, Ontario, Canada L8S $4 M 1$
}

(November 13, 2018)

\begin{abstract}
The electromagnetic response of a system with two planes per unit cell involves, in addition to the usual intraband contribution, an added interband term. These transitions affect the temperature dependence and the magnitude of the zero temperature $c$-axis penetration depth $\left(\lambda_{c}\right)$. When the interplane hopping $t_{\perp}$ is sufficiently small, the interband transitions dominate the low temperature behaviour of $\lambda(T)$ which then does not reflect the linear temperature dependence of the intraband term and in comparison becomes quite flat even for a $d$-wave gap. It is in this regime that the pseudogap was found in our previous normal state calculations of the $c$ axis conductivity, and the effects are connected.
\end{abstract}

74.25.Nf,74.25.Jb,74.72.-h

\section{INTRODUCTION}

Studies of the in plane low temperature penetration depth of single crystals of twinned and untwinned $\mathrm{YBa}_{2} \mathrm{Cu}_{3} \mathrm{O}_{x}\left(\mathrm{YBCO}_{x}\right)$ at optimum doping $\left(x \bar{T}_{6.93}\right)$ have revealed a linear temperature dependence.t5 This has been confirmed in more recent measurements on single crystals by Mao et al.6 and on thin films by de Vaulchier et al. 1 although reports of other power laws persist 8 Extensions to underdoped samples $\left(\mathrm{YBCO}_{6.6}\right)$ as well as to the overdoped case $\mathrm{YBCO}_{6.99}$ confirm that this linear law does not depend on oxygen doping although the value of its slope is affected. In untwinned single crystals, it has also been found that the zero temperature value of the in-plane penetration depth is highly anisotropic: $\lambda_{b}=1600 \AA$ while $\lambda_{a}=1030 \AA$ A were $b$ denotes the chain direction, and the sample doping is near optimal $\left(x=6.95\right.$ with $\left.T_{c}=93.2 \mathrm{~K}\right)$. This large anisotropy in $\lambda_{a b}(T=0)$ is, however, not reflected strongly in the temperature dependence of the normalized penetration depth $\lambda_{i}(0) / \lambda(T)$ which is found to be nearly the same for $i=a$ and $b, 0$ with that along the chain showing a slightly steeper slope.

A linear temperature behaviour is, of course, what is expected for a superconductor with a gap which has $d$ wave symmetry with zeros crossing the Fermi surface. Also, the addition of $\mathrm{Zn}$ impurities, 5 which are believed to act as unitary scatters, is found to alter the temperature dependence of $\lambda_{a b}$ from linear to $T^{2}$, which is expected for $d$-wave. There are now many other experiments that confirm the $d$-wave symmetry of the gap. Examples are angular resolved photoemission 113 (ARPES) as well as
SQUID 14 17 experiments. These latter experiments have been devised specifically to measure the phase of the gap rather than simply probe for zeros crossing the Fermi surface as does ARPES and penetration depth.

Detailed theoretical studies of the $a-b$ plane anisetropy have been carried out by the present author\$18 20 in a two plane model, coupled through a transverse tunnelling matrix element $\left(t_{\perp}\right)$. The general conclusion of these studies is that there is a significant amount of condensate on the chains and that the gap on the $\mathrm{CuO}$ chains must be of the same order of magnitude as on the $\mathrm{CuO}_{2}$ planes. The condensate on the chains is, of course, the ultimate source of the orthorhombicity which is reflected in the observed large in plane anisotropy for $\lambda_{a}$ and $\lambda_{b}$ at $\mathrm{T}=0$. The recent theoretical work of L'Donovan and Carbotte21 and of Xiang and Wheatley 22 further confirm these general conclusions. CITS23,24 experiments in which the gap is measured separately on the chains and on the plane give added evidence that both gap values are of roughly of the same size. An anisotropy $p$ similar magnitude is alspseen in the DC resistivity 2528 and optical conductivity 29 .

Recently, Hardy et al. 9 in addition to summarizing their result for $a-b$ plane anisotropy in the underdoped, optimally doped and gverdoped cases, have also presented $c$-axis data.9.10,30 For $\mathrm{YBCO}_{6.6}\left(T_{c}=59 \mathrm{~K}\right)$, they estimate $\lambda_{c} / \lambda_{a} \sim 31$. For $\mathrm{YBCO}_{6.95}\left(T_{c}=93.2 \mathrm{~K}\right)$ $\lambda_{c} / \lambda_{a} \sim 7$ which is about the same as for the overdoped case with $\mathrm{x}=6.99(\mathrm{~T}=89 \mathrm{~K})$. They give detailed results for the temperature variation of the ratio $\lambda_{c}(0) / \lambda_{c}(T)$ and find, in all cases, that at low $T$, the curve for the $c$-axis falls well above the linear dependence found for $\lambda_{a}$ and $\lambda_{b}$ and may vary as a higher than linear power. A striking fact is that the underdoped and overdoped case are found to have nearly the same dependence on reduced temperature $T / T_{c}$ while the optimally doped case falls slightly below. A possible explanation for the rather flat behaviour observed in the underdoped case is that it is representative of incoherent coupling between the chains and planes. This view is consistent with decreasing coupling between planar elements as oxygen is removed from the sample.

For fully oxygenated $\mathrm{YBCO}_{x}$, the experimental results on the $c$-axis DC resistivity now appear to converge. It is found that $\rho(T)$ is metallic in the $c$-direction and has a nearly linear temperature dependence.25.27.31 Further, the anisotropy ratio $\rho_{c} / \rho_{a b}$ is of order $30-70$ which is consistent with the value of $\lambda_{c} / \lambda_{a}$ quoted above if we assume that an effective mass model is qualitatively valid. On 
the other hand, when the sample is deoxygenated, the $c$-axis behaviour can change radically with the DC resistivity $\rho_{c}(T)$ showing a semiconducting-like behaviour at low temperature $\left(d \rho_{c} / d T<0\right)$.

Also, the ratio $\rho_{c} / \rho_{a b}$ is much bigger 32 34 in the underdoped samples thap for optimal doping. For example, at $T=100 \mathrm{~K}, \rho_{c} / \rho_{a b} 34$ is of order $10^{3}$. This large value is in accordance with the value found for the corresponding ratio of $\left(\lambda_{c} / \lambda_{b}\right)^{2}$. It is to be noted that as the temperature is increased, metallic behaviour is recovered even for $\mathrm{YBCO}_{6.6}$. The very large value of $\rho_{c}$ observed in the underdoped case and its semiconducting behaviour has been taken as evidence that the $c$-axis transport can not be described by conventional three dimensional coherent Bloch transport and that it is dominated by a different (incoherent) mechanism due to the highly two dimensional nature of the electronic states. Evidence for this is provided by estimates of the $c$-axis mean free path which find that it is of the order or less than the $c$-axis spacing.

Various possible mechanisms 35 bo for $c$-axis transport have been reviewed by $\mathrm{Zha}_{\mathrm{h}}$, Cooper and Pines.35 In particular, Rojo and Levin 38 have considered explicitly static and dynamic off diagonal disorders as well as the effect of the interplane matrix element $t_{\perp}$ and also include several limiting cases. In particular, ordinary transport and the pure incoherent case with $t_{\perp}=0$ and static impurity scattering are treated. This latter case has been discussed in detail in the work of Graf, Rainer and Sauls. 10 It leads to a $c$-axis resistivity that is inversely related to its in-plane value. The source of incoherent scattering in such models is, however, not clear. Another interesting idea is the dynamic thermal dephasing process suggested by Legget 39 in which in-plane thermal fluctuations can be larger than $t_{\perp}$ and so destroy coherent $c$-axis transport. This mechanism, however, cannot explain the observation that metallic transport is restored at high temperature for the underdoped samples. A further model based on resonant tunneling through a barrier layer which due to Abrikosov, 40 models the $c$-axis d.c. resistivity with some succes. Yet another approach assumes that the copper oxide planes cannot be described by Fermi liquid theory and spin and charge degrees of freedom are separated. 41 No consensus has yet emerged about $c$-axis coupling.

In this paper, we will be mainly interested in the $c$ axis penetration depth. In this case, Radtke, Kostur and Levin 42 have already given results for the $T=0$ value and temperature dependence of $\lambda_{c}(T)$ in a model which contains three distinct contributions dependent on $t_{\perp}$, a pure incoherent impurity assisted contribution as in the work of Graf et al 36 and a phonon assisted interlayer inelastic part. They find that when the direct contribution dominates, $\lambda_{c}$ and $\lambda_{a b}$ will have the same linear temperature dependence at low $T$ while for impurity assisted hopping $\lambda_{c}^{2}(T)$ will show a much flatter, more $s$-wave low temperature behaviour as is observed in experiments. There are two problems with this scenario as applied to underdoped YBCO. First, while $t_{\perp}$ may be small, the coherent part may not be negligible. Estimates of $t_{\perp}$ provided by Zha et al. .55 give $t_{\perp} \sim 3.0$ $\mathrm{meV}$ for $\mathrm{YBCO}_{6.68}$. This value is reduced by an order of magnitude from $t_{\perp} \sim 30-40 \mathrm{meV}$ estimated in optimally doped YBCO but is still significant and is certainly much larger than the value of $0.1 \mathrm{meV}$ estimated for $\mathrm{Bi}_{2} \mathrm{Sr}_{2} \mathrm{CaCu}_{2} \mathrm{O}_{8}$. Secondly, the impurity assisted contribution vanishes by symmetry for a $d$-wave superconductor if the impurity potential is isotropic in momentum space, i.e. a $\delta$-function in direct space. This may not be a serious obstacle, particularly in YBCO which is orthorhombic and so cannot have a purely $d$-wave order parameter. A more serious problem is the microscopic origin of this impurity scattering. This is particularly problematic since many experiments would lead us to believe that these crystals are in the clean limit as far as $a b$ plane properties are concerned.

In this paper, we study a different mechanism for the $c$ axis penetration depth. We consider the possibility that it is a manifestation of the interband transitions which become more important in the $c$-axis conductivity as $t_{\perp}$ is reduced in magnitude. In-previous work on the normal state A.C. conductivity 43 we found that the usual Drude-like intraband contribution is of order $t_{\perp}^{0}$ and the interband contribution goes like $t_{\perp}^{2}$ for the in-plane conductivity. On the other hand, for the $c$-axis conductivity, the intraband contribution goes like $t_{\perp}^{4}$ while the interband remains of order $t_{\perp}^{2}$. It is clear then that, for sufficiently small values of $t_{\perp}$, the interband contribution to the penetration depth will dominate, and $\lambda_{c}(T)$ may be significantly different from $\lambda_{a b}$. It is precisely for this case that we found a pseudo gap in the normal state $c$ axis conductivity. Thus the apperence of a pseudogap and a flat low temperature dependence in the c-axis penetration depth are connected in our theory.

In section II, we present the necessary formalism. Numerical results are given and discussed in section III. A brief conclusion is included in section IV.

\section{DERIVATIONS}

To remain simple, we consider two isolated planes coupled with a transverse matrix element $t_{\perp}$. The uncoupled planes have dispersion

$$
\xi_{1}=-2 \sigma_{1}\left[\cos \left(k_{x}\right)+\cos \left(k_{y}\right)-2 B \cos \left(k_{x}\right) \cos \left(k_{y}\right)\right]-\mu_{1},
$$

representing the tetragonal $\mathrm{CuO}_{2}$ plane and

$$
\xi_{2}=-2 \sigma_{2} \cos \left(k_{y}\right)-\mu_{2}
$$

representing the orthorhombic $\mathrm{CuO}$ chains. In (11) and (2), $8 \sigma_{1}$ and $4 \sigma_{2}$ are the band widths, $B$ is a next nearest neighbour hopping amplitude in units $\sigma_{1}$, and $\mu_{1}$ and $\mu_{2}$ are chemical potentials related to the fillings on the 
chains and planes. This model is already quite complicated to deal with and final results are obtained only numerically. Nevertheless, it needs to be recognized that it is still oversimplified and may not apply directly to YBCO. For instance, in YBCO, there are three planes per unit cell: a bilayer of $\mathrm{CuO}_{2}$ planes and a layer of $\mathrm{CuO}$ chains in between them. These 3 planes form the basic unit cell which is then coupled to the other unit cells through additional, presumably insulating, layers. Under such circumstances, estimates of the transverse coupling $t_{\perp}$ provided from DC resistivity data may well reflect the value of the intercell coupling (which could be incoherent) rather than the plane-chain coupling within the cell (which could be coherent). Treating such complications is, however, beyond the scope of this paper. Here, we will assume only 2 planes but take one to be chains so as to introduce the observed orthorhombicity. The transverse coupling $t_{\perp}$ therefore represents both the chain-plane coupling and the intercell coupling.

The mean field Hamiltonian for our model is

$$
H=\sum_{\mathbf{k}} C_{\mathbf{k}}^{\dagger} h(\mathbf{k}) C_{\mathbf{k}}
$$

where $C_{\mathbf{k}}^{\dagger}=\left[c_{1 \mathbf{k} \uparrow}^{\dagger}, c_{1-\mathbf{k} \downarrow}, c_{2 \mathbf{k} \uparrow}^{\dagger}, c_{2-\mathbf{k} \downarrow}\right]$ and $c_{i \mathbf{k} \sigma}^{\dagger}$ creates an electron of spin $\sigma$ and momentum $\mathbf{k}$ in the plane $(i=1)$ or chain $(i=2)$ layers. The Hamiltonian matrix is

$$
h(\mathbf{k})=\left[\begin{array}{cccc}
\xi_{1} & \Delta_{\mathbf{k}} & t(\mathbf{k}) & 0 \\
\Delta_{\mathbf{k}} & -\xi_{1} & 0 & -t(\mathbf{k}) \\
t(\mathbf{k}) & 0 & \xi_{2} & \Delta_{\mathbf{k}} \\
0 & -t(\mathbf{k}) & \Delta_{\mathbf{k}} & -\xi_{2}
\end{array}\right]
$$

In Eq. (4) $t(\mathbf{k})=-2 t_{\perp} \cos \left(k_{z} d / 2\right)$, which follows from a tight-binding model of $c$-axis coupling. The unit cell size is $d$ along the $c$-axis. The superconducting gap $\Delta_{\mathbf{k}}$ is chosen to be the same in both the plane and chain layers. We will not determine $\Delta_{\mathbf{k}}$ through solution of a gap equation but will instead be guided by experiments which have indicated that the gap on the planes and chains are very similar. For our simple model we have a single $d$ wave gap characteristic of the entire system. We make no attempt to relate this gap to the basic microscopic interactions but simply postulate its existence.

In a previous paper, we derived a general expression for the penetration depth of a system with two atoms per unit cell (where the two atoms form the chain and plane layers).20 The expression for the penetration depth required the two particle Green's function:

$$
\begin{aligned}
G_{\mu \nu}\left(\mathbf{q}, \mathbf{q}^{\prime}, \omega\right)= & -\delta_{\mathbf{q}, \mathbf{q}^{\prime}} \frac{e^{2}}{\Omega} \sum_{i, j, \mathbf{k}}\left[\hat{\gamma}_{\mu}(\mathbf{k}, \mathbf{k}+\mathbf{q})\right]_{i j}\left[\hat{\gamma}_{\mu}(\mathbf{k}+\mathbf{q}, \mathbf{k})\right]_{j i} \\
& \times \frac{f\left[E_{i}(\mathbf{k})\right]-f\left[E_{j}(\mathbf{k}+\mathbf{q})\right]}{E_{i}(\mathbf{k})-\hbar \omega-E_{j}(\mathbf{k}+\mathbf{q})}
\end{aligned}
$$

where $f(x)$ is the Fermi distribution function, $e$ is the electronic charge and the sum over $i$ and $j$ range from 1 to 4 . In (5), the electromagnetic vertex $\hat{\gamma}_{\mu}(\mathbf{k}+\mathbf{q}, \mathbf{k})$ is a four by four matrix related to the bare vertex function $\gamma_{\mu}(\mathbf{k}+\mathbf{q}, \mathbf{k})$ through the unitary transformation $U(\mathbf{k})$ which diagonalizes the Hamiltonian. That is

$$
\hat{\gamma}_{\mu}(\mathbf{k}, \mathbf{k}+\mathbf{q})=U^{\dagger}(\mathbf{k}) \gamma_{\mu}(\mathbf{k}, \mathbf{k}+\mathbf{q}) U(\mathbf{k}+\mathbf{q}) .
$$

The quasiparticle energies $E_{i}(\mathbf{k})$ are the eigenvalues of $h(\mathbf{k}): E_{1}=E_{+}, E_{2}=-E_{+}, E_{3}=E_{-}, E_{4}=-E_{-}$, where

$$
E_{ \pm}=\sqrt{\epsilon_{ \pm}^{2}+\Delta_{\mathbf{k}}^{2}}
$$

and $\epsilon_{ \pm}$are the normal state band energies

$$
\epsilon_{ \pm}=\frac{\xi_{1}+\xi_{2}}{2} \pm \sqrt{\left[\frac{\xi_{1}-\xi_{2}}{2}\right]^{2}+t^{2}} .
$$

For a static external electromagnetic vector potential $A(\mathbf{r})$, the current is given by

$$
J_{\mu}(\mathbf{r})=-\sum_{\nu} K_{\mu \nu} A_{\nu}(\mathbf{r})
$$

where $(\mu, \nu)$ indicates spatial directions and the kernel $K_{\mu \nu}$ is given by

$$
K_{\mu \nu}=\frac{1}{c}\left\{\left.G_{\mu \nu}(0,0,0)\right|_{\Delta=0}-G_{\mu \nu}(0,0,0)\right\} .
$$

In equation (10), $c$ is the velocity of light, $\Omega$ is the volume and the first term in the curly bracket is the same as the second except that it is to be evaluated in the normal state with gap set equal to zero. Note that only the $\mathbf{q}=\mathbf{k}=0$ and $\omega=0$ limit has been taken in (10). This is sufficient for calculations of the penetration depth which follows from

$$
\frac{1}{\lambda_{\mu}^{2}(T)}=\frac{c}{4 \pi} K_{\mu \mu}
$$

Our fundamental formula therefore, has the form

$$
\begin{aligned}
G_{\mu \mu}(0,0,0) & =-\frac{e^{2}}{\Omega} \sum_{i, j, \mathbf{k}}\left[\hat{\gamma}_{\mu}(\mathbf{k}, \mathbf{k})\right]_{i j}^{2} \\
& \times\left[\delta_{i j} \frac{\partial f\left(E_{i}\right)}{\partial E_{i}}-\left[1-\delta_{i j}\right] \frac{f\left(E_{i}\right)-f\left(E_{j}\right)}{E_{i}-E_{j}}\right] .
\end{aligned}
$$

The matrix $U(\mathbf{k})$ which diagonalizes the Hamiltonian matrix can be found using a two step process. We first introduce a unitary matrix $U_{N}$ which would diagonalize the normal state alone. It has the form

$$
U_{N}=\left[\begin{array}{cccc}
u_{n} & 0 & v_{n} & 0 \\
0 & u_{n} & 0 & v_{n} \\
v_{n} & 0 & -u_{n} & 0 \\
0 & v_{n} & 0 & -u_{n}
\end{array}\right]
$$

with 


$$
u_{n}=\sqrt{\frac{\xi_{1}-\epsilon_{-}}{\epsilon_{+}-\epsilon_{-}}}
$$

and

$$
v_{n}=\sqrt{\frac{\epsilon_{+}-\xi_{1}}{\epsilon_{+}-\epsilon_{-}} .}
$$

Then

$$
\begin{aligned}
h^{\prime} & =U_{N}^{\dagger} h U_{N} \\
& =\left[\begin{array}{cccc}
\epsilon_{+} & \Delta_{\mathbf{k}} & 0 & 0 \\
\Delta_{\mathbf{k}} & -\epsilon_{+} & 0 & 0 \\
0 & 0 & \epsilon_{-} & \Delta_{\mathbf{k}} \\
0 & 0 & \Delta_{\mathbf{k}} & -\epsilon_{-}
\end{array}\right]
\end{aligned}
$$

Matrix (16) can finally be diagonalized by application of a second unitary transformation $U_{S}$ to get

$$
E_{i}=\left[U_{S}^{\dagger} h^{\prime} U_{S}\right]_{i i}
$$

where

$$
U_{S}=\left[\begin{array}{cccc}
u_{+} & v_{+} & 0 & 0 \\
v_{+} & -u_{+} & 0 & 0 \\
0 & 0 & u_{-} & v_{-} \\
0 & 0 & v_{-} & -u_{-}
\end{array}\right]
$$

with

$$
u_{ \pm}=\sqrt{\frac{E_{ \pm}+\epsilon_{ \pm}}{2 E_{ \pm}}}
$$

and

$$
v_{ \pm}=\sqrt{\frac{E_{ \pm}-\epsilon_{ \pm}}{2 E_{ \pm}}} .
$$

where we stress again, a single gap has been introduced for the condensate on both the $\mathrm{CuO}_{2}$ planes and $\mathrm{CuO}$ chains equally. This assumption was justified in our previous work 20 and has simplified the mathematics enormously here.

There remains only to compute the transformed electromagnetic vertex $\hat{\gamma}=U_{S}^{\dagger} U_{N}^{\dagger} \gamma U_{N} U_{S}$ which appears in equation (12). The bare vertices are 20

$$
\begin{gathered}
\gamma_{x}=\left[\begin{array}{cccc}
v_{1 x} & 0 & 0 & 0 \\
0 & v_{1 x} & 0 & 0 \\
0 & 0 & 0 & 0 \\
0 & 0 & 0 & 0
\end{array}\right], \\
\gamma_{y}=\left[\begin{array}{cccc}
v_{1 y} & 0 & 0 & 0 \\
0 & v_{1 y} & 0 & 0 \\
0 & 0 & v_{2 y} & 0 \\
0 & 0 & 0 & v_{2 y}
\end{array}\right],
\end{gathered}
$$

$$
\gamma_{z}=\left[\begin{array}{cccc}
0 & 0 & v_{\perp} & 0 \\
0 & 0 & 0 & v_{\perp} \\
v_{\perp} & 0 & 0 & 0 \\
0 & v_{\perp} & 0 & 0
\end{array}\right]
$$

with

$$
v_{i \mu}=\frac{1}{\hbar} \frac{\partial h_{i i}}{\partial k_{\mu}}
$$

and

$$
v_{\perp}=\frac{1}{\hbar} \frac{\partial t}{\partial k_{z}} .
$$

It is straightforward to show that

$$
\hat{\gamma}=\left[\begin{array}{cccc}
v_{\mu+} & 0 & \alpha v_{\mu} & -\beta v_{\mu} \\
0 & v_{\mu+} & \beta v_{\mu} & \alpha v_{\mu} \\
\alpha v_{\mu} & \beta v_{\mu} & v_{\mu-} & 0 \\
-\beta v_{\mu} & \alpha v_{\mu} & 0 & v_{\mu-}
\end{array}\right]
$$

where

$$
v_{\mu \pm}=\frac{1}{\hbar} \frac{\partial \epsilon_{ \pm}}{\partial k_{\mu}}
$$

and

$$
v_{x}=\frac{t}{\epsilon_{+}-\epsilon_{-}} v_{1 x}
$$

$$
\begin{gathered}
v_{y}=\frac{t}{\epsilon_{+}-\epsilon_{-}}\left(v_{1 y}+v_{2 y}\right), \\
v_{z}=\frac{\xi_{2}-\xi_{1}}{\epsilon_{+}-\epsilon_{-}} v_{\perp} .
\end{gathered}
$$

The coherence factors $\alpha$ and $\beta$ have the simple form

$$
\begin{aligned}
& \alpha^{2}=\frac{1}{2} \frac{E_{+} E_{-}+\epsilon_{+} \epsilon_{-}+\Delta^{2}}{E_{+} E_{-}} \\
& \beta^{2}=\frac{1}{2} \frac{E_{+} E_{-}-\epsilon_{+} \epsilon_{-}-\Delta^{2}}{E_{+} E_{-}} .
\end{aligned}
$$

Now that $\hat{\gamma}$ has been specified, $G_{\mu \mu}$ may be evaluated.

\section{RESULTS AND DISCUSSION}

We start by rewriting equation (12) (which gives the penetration depth $\lambda(T)$ through (10) and (11)) in the form

$$
\begin{aligned}
G_{\mu \mu}(0,0,0)= & -\frac{e^{2}}{\Omega} \sum_{\mathbf{k}}\left\{\sum_{i}\left[\hat{\gamma}_{\mu}\right]_{i i}^{2} \frac{\partial f\left(E_{i}\right)}{\partial E_{i}}\right. \\
& \left.-\sum_{i \neq j}\left[\hat{\gamma}_{\mu}\right]_{i j}^{2} \frac{f\left(E_{i}\right)-f\left(E_{j}\right)}{E_{i}-E_{j}}\right\}
\end{aligned}
$$


The first term in 30 is the very familiar intraband contribution and can be rewritten to read

$$
-2 \frac{e^{2}}{\Omega} \sum_{\mathbf{k}}\left\{\left[\frac{\partial \epsilon_{+}}{\partial k_{\mu}}\right]^{2} \frac{\partial f\left(E_{+}\right)}{\partial E_{+}}-\left[\frac{\partial \epsilon_{-}}{\partial k_{\mu}}\right]^{2} \frac{\partial f\left(E_{-}\right)}{\partial E_{-}}\right\}
$$

which is just the usual expression for the penetration depth in a 2 band model with energies given by equation (8). For $t_{\perp}=0$ equation (31) will make no contribution to $\lambda_{z}$ and we recover the result for 2 decoupled bands. It is to be noted that the second term in (30), which is new and which gives the interband transitions, will also give no contribution to $\lambda_{z}$ when $t_{\perp}=0$ as it must. This can be seen by noting that each of the vertex elements $\left[\hat{\gamma}_{\mu}\right]_{i j}$, with $i \neq j$, is proportional to $t_{\perp}$. The interband contribution to coming from the second term in (30) can be written as

$$
\begin{array}{r}
4 \frac{e^{2}}{\Omega} \sum_{\mathbf{k}} v_{\mu}^{2}\left\{\alpha^{2} \frac{f\left(E_{+}\right)-f\left(E_{-}\right)}{E_{+}-E_{-}}\right. \\
\left.-\beta^{2} \frac{1-f\left(E_{+}\right)-f\left(E_{-}\right)}{E_{+}+E_{-}}\right\}
\end{array}
$$

where $\alpha$ and $\beta$ are given by Eqs. (29a) and (29b). These terms describe interband transitions. The term with $\left[f\left(E_{+}\right)-f\left(E_{-}\right)\right] /\left(E_{+}-E_{-}\right)$vanishes at $T=0$ and increases with increasing $T$ while the one with $\left[1-f\left(E_{+}\right)-f\left(E_{-}\right)\right] /\left(E_{+}+E_{-}\right)$is 1 at $T=0$ and decreases with increasing $T$. This competition between these two terms leads to a much flatter temperature dependence for $\lambda^{-2}(T)$ than does the more common intraband term (31). Choosing the $z$ direction, we note that the intraband part (31) goes like $t^{4}$ because $v_{z}$ in $(28 \mathrm{~d})$ goes like $t_{\perp}^{2}$ while the interband contribution 32 goes only like $t_{\perp}^{2}$. For sufficiently small values of $t_{\perp}$, it is clear that the interband contribution will dominate the $c$-axis penetration depth and that our intuition based only on the intraband band term will no longer hold. Our numerical work, to be described below, will tell us the value of $t_{\perp}$ where this new regime of behaviour is reached. Before presenting results it is interesting to note that the in-plane situation is quite different with regards to the dominance, or lack there of, of the interband processes. This can be seen from equation (31) and the realization that, for the $\mathrm{x}$ or $\mathrm{y}$ directions, the intraband contribution is, to leading order, independent of $t_{\perp}$, while from (32), we see that the interband part is proportional to $t_{\perp}^{2}$. Thus, for small $t_{\perp}$, the interband contribution to the in-plane penetration depth will not be important compared with the intraband contribution and the familiar behaviour will result. On the other hand, when $t_{\perp}$ gets large, the interband contribution to the in-plane penetration depth can become of some importance.

In Fig. 1, we show our model band structure. We have taken $\left\{\sigma_{1}, \sigma_{2}, \mu_{1}, \mu_{2}, t_{\perp}\right\}=\{70,100,-65,-175,20\} \mathrm{meV}$ with $B=0.45$. The dashed line gives the plane and chain Fermi surface contour in the 2-dimensional $\mathrm{CuO}_{2}$ plane Brillouin zone as a function of momentum components

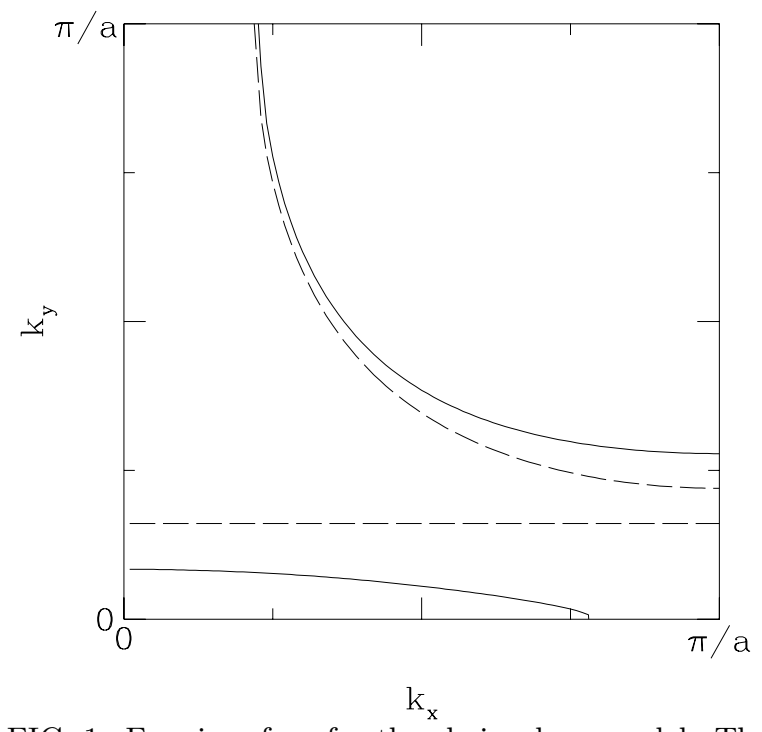

FIG. 1. Fermi surface for the chain-plane model. The param-

eters are $\left\{\sigma_{1}, \sigma_{2}, \mu_{1}, \mu_{2}, t_{\perp}\right\}=\{70,100,-65,-175,20\} \mathrm{meV}$ and $B=0.45$. The dashed lines apply to the unperturbed case $t_{\perp}=0$ and represent uncoupled plane and chain layers. They also describe the case $k_{z}=\pi / d$ in equation (6), i.e. the Brillouin zone boundary. The solid curves are for $k_{z}=0$ in (6), i.e. the central plane in the B.Z. while the area between solid and dashed curves represent the dispersion in the z-direction.

$k_{x}$ and $k_{y}$. When the transverse matrix element $t_{\perp}$ is taken into account, the Fermi surface acquires dispersion in the $z$-direction. When $k_{z}$ is zero, $t\left(k_{z}\right)=2 t$ and we get the hybridized Fermi surface shown as the solid contours in Fig. 1. On the other hand, for $k_{z}$ at the zone edge $\left(k_{z}=\pi / d\right), t\left(k_{z}\right)=0$ and we recover the dashed contours. For other values of $k_{z}$, we obtain Fermi surface contours intermediate between solid and dashed curve and the area between these 2 contours gives the $z$ dispersion of our model. This simplified Fermi surface will be kept fixed in our calculations and we present results for the penetration depth as a function of temperature for all three directions $x, y, z$ (or, equivalently $a, b, c$ ).

What is given in Figs. 2, 3 and 4 are the temperature dependences of $\left[\lambda_{\mu}(0) / \lambda_{\mu}(T)\right]^{2}$ for $t_{\perp}=20 \mathrm{meV}, 10 \mathrm{meV}$ and $5 \mathrm{meV}$, respectively. In all cases, we have used a $d$-wave gap of the form

$$
\Delta_{\mathbf{k}}=\Delta_{0}\left[\cos \left(k_{x}\right)-\cos \left(k_{y}\right)\right]
$$

over the 2-dimensional Brillouin zone with $\Delta_{0}(T)$ taken to have a BCS temperature variation but scaled so that $2 \Delta_{0}(T=0) / k_{B} T_{c}=7$. This value is chosen to agree with experiments on $\mathrm{YBCO}$ which find a rather large value for the gap to critical temperature ratio. This is also necessary in order to get an in-plane temperature dependence 


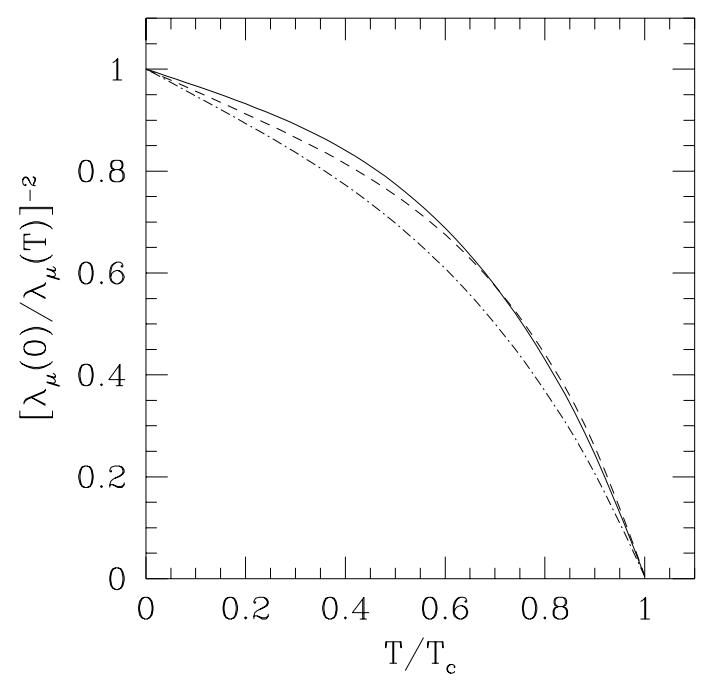

FIG. 2. The inverse square penetration depth normalized to its value at $T=0$ in the a (dashed curve), b (dot-dashed curve) and c (solid curve) directions as a function of reduced temperature $T / T_{c}$. The transverse matrix element coupling the chain and plane layers is $t_{\perp}=20 \mathrm{meV}$.

for the penetration depth which is in reasonable accord with experiment. Before describing our results and commenting on them, we stress that in $\mathrm{YBCO}_{x}$ as a function of oxygen doping, the Fermi surface of the chains will certainly be changed in important ways because oxygen depletion occurs on the chains as we go from the overdoped case $x=7$ to the underdoped case with $x=6.6$. At the same time, the number of holes on the planes as well as on the chains will change so the value of the chemical potential and displacement between bands, $\mu_{1}$ and $\mu_{2}$ will change as will the filling factor in each band. Such details are not yet known and in the absence of such information, we have chosen to keep the bands fixed as well as the filling which was not chosen to specifically model the overdoped or underdoped case. Here, we are primarily interested in understanding the effect of interband transitions on the penetration depth and their variation with chain-plane coupling $t_{\perp}$ which may change significantly with doping although again this is not well known.

In Figs. 2 to 4 , the dashed curve is for the $a$-direction, the dot-dashed curve is for the $b$-direction and the solid for the $c$-direction. In the first case, $t_{\perp}=20 \mathrm{meV}$ and all three directions show about the same temperature dependence with $\lambda_{b}^{-2}$ having the steepest slope at low $T, \lambda_{a}^{-2}$ having a slightly flatter slope and $\lambda_{c}^{-2}$ having the flattest slope. This order is as observed in experiment. As the transverse hopping matrix element $t_{\perp}$ is reduced in size, we see that the in plane behaviour is not strongly affected but the $c$-axis is. It becomes flatter at low temperature and begins to look much more like a usual $s$-wave case.

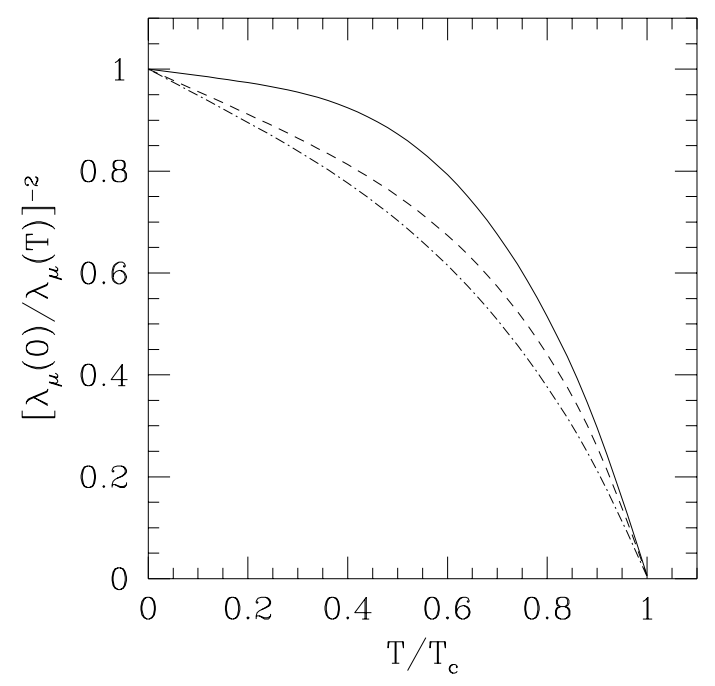

FIG. 3. As in Fig. 2, penetration depth in the a (dashed curve), b (dot-dashed curve) and c (solid curve) directions but with $t_{\perp}=10 \mathrm{meV}$.

This different behaviour results when the interband terms (32) start becoming dominant over the more conventional intraband term (31). We might expect this to occur in the underdoped situation in YBCO where $t_{\perp}$ is expected to be of order $5 \mathrm{meV}$ or so. For optimally doped YBCO, interband terms contribute less to the $c$-axis behaviour and the system becomes more three dimensional.

In Fig. 5, we show the absolute value of the inverse square of the zero temperature penetration depth $\lambda(0)^{-2}$ for the three directions. Note the difference in scale between $\lambda_{c}^{-2}$ and $\lambda_{a b}^{-2}$. In the plot of $\lambda_{c}(0)$, we can see very clearly the $t_{\perp}^{2}$ dependence discussed earlier. The magnitude of $\lambda_{a}^{-2}$ is smaller than $\lambda_{b}^{-2}$, roughly by a factor of two because of the additional screening current carried by the chains. The difference is reduced as $t_{\perp}$ increases. This is attributed to the increase significance of the interband contribution to the $b$-direction penetration depth as $t_{\perp}$ is increased. In the $\mathrm{x}$ direction, the intraband term in (31) goes like

$$
v_{1 x}^{2}\left[1+O\left(\frac{t^{2}}{\left(\xi_{1}-\xi_{2}\right)^{2}}\right)\right]
$$

for most of the Brillouin zone and the second term is small as is the interband term (32) which also varies as $O\left(t^{2} /\left(\xi_{1}-\xi_{2}\right)^{2}\right)$. In the region where the plane and chain Fermi surface crosses this argument does not apply, but $v_{1 x}^{2}$ is small there and so we can conclude that the terms in $t_{\perp}$ are not important. This does not hold, however, for the y-direction because in that case $v_{1 y}$ and $v_{2 y}$ are not necessarily small in regions of the Brillouin zone where the corrections in $t_{\perp}$ are significant and we expect some contribution to $\lambda_{y}^{-2}(T)$. 


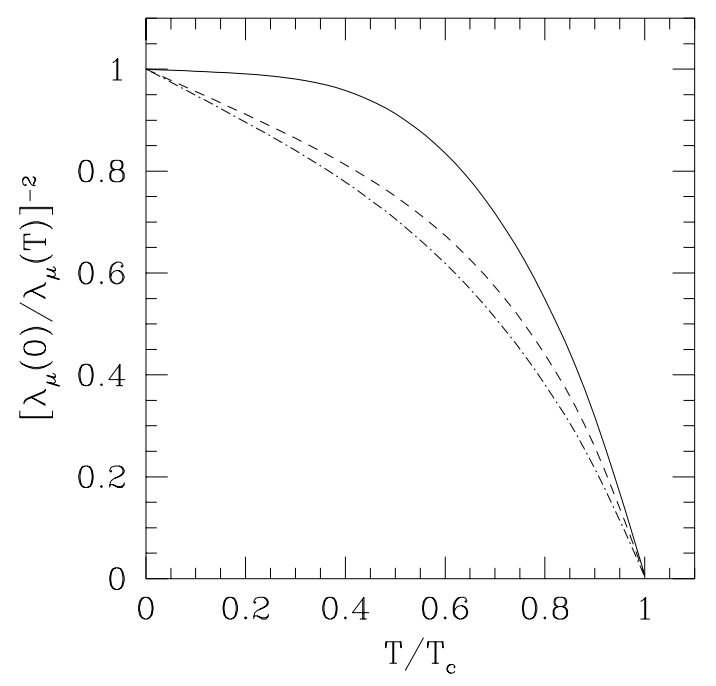

FIG. 4. As in Fig. 2, penetration depth in the a (dashed curve), b (dot-dashed curve) and c (solid curve) directions but with $t_{\perp}=5 \mathrm{meV}$.

\section{CONCLUSION}

We have considered a model of two conducting planes per unit cell, one with tetragonal symmetry representing a $\mathrm{CuO}_{2}$ plane, the other with orthorhombic symmetry, representing a $\mathrm{CuO}$ chain. A transverse hopping matrix element $t_{\perp}$ is included which couples the two bands coherently and introduces the third dimension. For such a model, new transitions enter the electromagnetic properties that would not be present in the case of one atom per unit cell. In addition to the usual intraband terms, there are terms which now correspond to interband transitions. We have investigated the effect of these terms on the value of the zero temperature penetration depth and on its temperature dependence. It is found that for the $a$-direction, the effects of $t_{\perp}$ are small while for the $b$-direction, along the chains, there can be significant corrections to the intraband terms, although to leading order $\lambda_{b}$ is still independent of $t_{\perp}$. In this case, the corrections for the interband terms go like $t_{\perp}^{2}$.

For the $c$-direction (perpendicular to the planes), the situation is quite different. The conventional intraband terms go like $t_{\perp}^{4}$ while the interband ones go like $t_{\perp}^{2}$ so, for sufficiently small values of $t_{\perp}$, the interband contribution eventually must take over. We find that these have a new temperature dependence quite distinct from the linear law expected for a $d$-wave superconductor at low temperature. They give a much flatter curve at low temperature for the $c$-axis superfluid density. Numerical calculations indicate that the interband term will be important when $t_{\perp}$ is of order $5-10 \mathrm{meV}$. This value is reasonable for underdoped YBCO. In our previous work

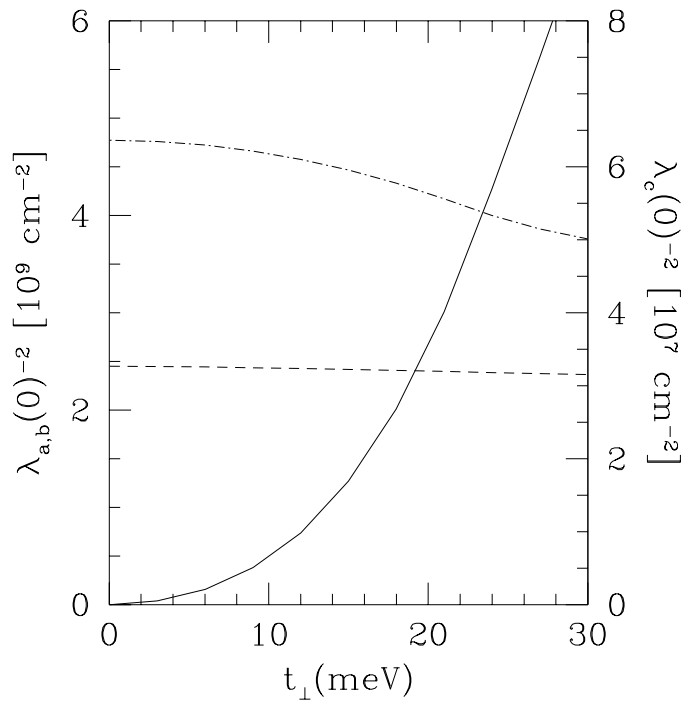

FIG. 5. Magnitude of the inverse penetration depth at $T=0$ as a function of the plane-chain coupling. The three curves are $\lambda_{a}(0)$ (dashed), $\lambda_{b}(0)$ (dot-dashed) and $\lambda_{c}(0)$ (solid).

on the normal state, we found that a pseudogap develops in the $c$ axis optical conductivity for such small values of $t_{\perp}$. This offers an alternate interpretation of the $c$ axis data in the underdoped case, which does not rely on any incoherent elastic scattering or $c$-axis out-of-plane phonon assisted process. Our calculations naturally give a linear dependence in $a$ - and $b$ - directions and a much flatter dependence for the $c$-axis as is observed. On the other hand, as the coupling between the two planes is increased, the system becomes much more 3 dimensional and the temperature dependence of all three penetration depths become similar and are linear at low $\mathrm{T}$ for a $d$ wave gap. These findings are all in general qualitative agreement with the data in YBCO.

As discussed in the text, however, our results can probably not be applied to the oxides without modifications because our electronic structure model is too simple. For example, the unit cell in $\mathrm{YBCO}$ contains a $\mathrm{CuO}_{2}$ bilayer as well as $\mathrm{CuO}$ chains. The coupling between planes within a cell is likely to be quite different from that between two cells while in our simplified model a single $t_{\perp}$ enters. Still, the model allows us to introduce and understand better the role that interband transitions can play in the $c$-axis penetration depth. It exhibits many of the qualitative properties observed in YBCO.

\section{ACKNOWLEDGEMENTS}

Research supported in part by the Canadian Institute for Advanced Research (CIAR) and by the Natural 
Sciences and Engineering Research Council of Canada (NSERC).

$\dagger$ Present Address: Dept. of Physics, Indiana University, Swain Hall W 117, Bloomington IN 47405.

${ }^{1}$ W.N. Hardy et al., Phys. Rev. Lett. 70, 3999 (1993).

${ }^{2}$ D.A. Bonn, P. Dosanjh, R. Liang and W.N. Hardy, Phys. Rev. Lett. 68, 2390 (1992).

${ }^{3}$ K. Zhang et al., Phys. Rev. 73, 2484 (1994).

${ }^{4}$ D.A. Bonn et al., J. Phys. Chem. Solids 56, 1941 (1995).

${ }^{5}$ D.A. Bonn, S. Kumal et al., Phys. Rev. B 50, 405 (1994).

${ }^{6}$ Mao et al., Phys. Rev. B 51, 3316 (1995).

${ }^{7}$ L.A. de Vaulchier et al., Europhys. Lett. 33, 153 (1996).

${ }^{8}$ A. Fuchs, W. Prusseit, P. Berlerich and H. Kinder, Phys. Rev. B 53, R14745 (1996).

${ }^{9}$ W.N. Hardy, S. Kamal, Ruixing Liang, D.A. Bonn, C.C. Homes, D.N. Basov and T. Timusk (Houston Workshop 1996).

${ }^{10}$ D.A. Bonn, S. Kamal, K. Zhang, R. Liang and W.N. Hardy, J. Phys. Chem. Solids 56, 1941 (1995).

${ }^{11}$ C.G. Olson et al., Science 245, 731 (1989).

12 Z.X. Shen et al., Phys. Rev. Lett. 70, 1553 (1993).

${ }^{13}$ H. Ding, J.C. Campuzano et al., Phys. Rev. Lett. 50, 1333 (1994).

${ }^{14}$ D.A. Wollman, D.J. Van Harlingen, W.C. Lee, D.M. Ginsberg and A.J. Leggett, Phys. Rev. Lett. 71, 2134 (1993).

15 C.C. Tsuei et al., Phys. Rev. Lett. 73, 593 (1994).

16 J.R. Kirtley, C.C. Tsuei, J.Z. Sun, C.C. Chi, Lock See YuJahnes, A. Gupta, M. Rupp and M.B. Ketchen, Nature 373, 225 (1995).

${ }^{17}$ C.C. Tsuei et al., Science 271, 329 (1996).

${ }^{18}$ W.A. Atkinson and J.P. Carbotte, Phys. Rev. B 511161 (1995); Phys. Rev. B 51, 16371 (1995).

19 W.A. Atkinson and J.P. Carbotte, Phys. Rev. B 52, 6894 (1995).

${ }^{20}$ W.A. Atkinson and J.P. Carbotte, Phys. Rev. B 52, 10601 (1995).

${ }^{21}$ C. O'Donovan and J.P. Carbotte, Phys. Rev. B (in press).

${ }^{22}$ T. Xiang and J.M. Wheatley, Phys. Rev. Lett. 76, 134 (1996).

${ }^{23}$ H.L. Edwards, J.T. Markert and A.L. de Lozanne, Phys. Rev. Lett. 73, 2967 (1992).

${ }^{24}$ H.L. Edwards, D.J. Derro, A.L. Barr, J.T. Markert and A.L. de Lozanne, Phys. Rev. Lett. 75, 1387 (1992).

${ }^{25}$ T.A. Friedmann, M.w. Rabin, J. Giapintzakis, J.P. Rice and D.M. Ginsberg, Phys. Rev. B 42, 6217 (1990).

${ }^{26}$ U. Welp, S. Fleshler, W.K. Kwok, J. Downey, Y. Fang, G.W. Crabtree and J.Z. Liu, Phys. Rev. B 42, 10189 (1990).

27 Y. Iye in Physical Properties of High Temperature Superconductors, Vol. III (edited by D.M. Ginsberg, World Scientific, Singapore (1992)), p. 285.

${ }^{28}$ R. Gagnon, C. Lupien and L. Taillefer, Phys. Rev. B 50, 3458 (1994).

${ }^{29}$ D.N. Basov, R. Liang, D.A. Bonn, W.N. Hardy, B.
Dabrowski, M. Quijada, D.B. Tanner, J.P. Rice, D.M. Ginsberg and T. Timusk, Phys. Rev. Lett. 74, 598 (1995).

${ }^{30}$ C.C. Homes, T. Timusk, D.A. Bonn, R. Liang and W.N. Hardy, Physica C 254, 265 (1995).

${ }^{31}$ H. Takei, H. Asaoka, Y. Iye and H. Takeya, Jpn. J. Appl. Phys. 30, L1102 (1991).

${ }^{32}$ Y. Iye, T. Tamegai, T. Sakakibara, T. Goto, N. Miura, H. Takaya and H. Takei, Physica C 153-155, 26 (1988).

33 D.A. Brawner, Z.Z. Wang and N.P. Ong, Phys. Rev. B 40, 9329 (1989).

${ }^{34}$ K. Takenaka, K. Mizuhashi, H. Takagi and S. Uchida, Phys. Rev. B 50, 6534 (1994).

${ }^{35}$ Y. Zha, S.L. Cooper and D. Pines, Phys. Rev. B 53, 8253 (1996).

${ }^{36}$ M.J. Graf, D. Rainer and J.A. Sauls, Phys. Rev. B 47, 12089 (1993).

${ }^{37}$ N. Kumar and A.M. Jayannavar, Phys. Rev. B 45, 5001 (1992).

38 A.G. Rojo and K. Levin, Phys. Rev. B 48, 1686 (1993).

39 A.J. Leggett, Braz. J. Phys. 22, 129 (1992).

${ }^{40}$ A. A. Abrikosov, Physica C 258, 53 (1996).

${ }^{41}$ P. W. Anderson, Science 268, 1154 (1995), and references contained therein.

${ }^{42}$ R.J. Radtke, V.N. Kostur and K. Levin, Phys. Rev. B 53, R522 (1996).

${ }^{43}$ W. A. Atkinson and J. P. Carbotte, Phys. Rev. B (In Press); cond-mat/9608137. 